\title{
New Trend in Neuromyelitis Optica Spectrum Disorder (NMOSD)
}

\author{
Adel Ekladious ${ }^{1,2 *}$ \\ ${ }^{1}$ Associate professor of medicine, Faculty of health and medical sciences, University of Western Australia, 35/ Stirling Highway, Perth \\ Western Australia \\ ${ }^{2}$ Royal Hobart Hospital, 48 Liverpool Street, Hobart TAS 7000 \\ *Corresponding Author: Adel Ekladious, Associate professor of medicine, Faculty of health and medical sciences, University of \\ Western Australia, 35/ Stirling Highway, Perth Western, Royal Hobart Hospital, 48 Liverpool Street, Hobart TAS 7000, Australia. \\ Ph No: 061499449905
}

Received: January 31, 2022; Published: February 10, 2022

DOI: $10.55162 /$ MCMS.02.020

\begin{abstract}
NMSOD is an inflammatory disease affecting the optic nerves and spinal cord. It is an uncommon disease but is a differential that presents similarly to a few common diseases. Misdiagnosing NMOSD for multiple sclerosis might result in severe health consequences and the rapid deterioration of the patient if they are treated with disease modifying agents like interferon beta, fingolimod or natalizumab. It is noted that this disease affects females predominantly.
\end{abstract}

NMOSD is characterized by a disruption of the blood brain barrier during flares, and commonly involves the dorsal medulla, brain stem, cerebrum, and cerebellum. Involvement of the putamen is quite specific for the disease. In addition, Aquaporin-4 antibody is a NMOSD specific autoantibody which is rarely found in other diseases or healthy persons. In rare cases, Aquaporin-4 may not be detected in the disease, resulting in a variant that has been designated Aquaporin-4 negative NMOSD. Seropositive NMSOD commonly manifests as bilateral optic neuritis and longitudinal extensive myelitis. Before the confirmation of a NMSOD diagnosis, other differentials that present with bilateral optic neuritis and long extensive transverse myelitis should be ruled out [1].

International consensus criteria in 2015 adapted a broader criteria for the diagnosis of NMOSD which advised that in order to diagnose NMOSD, patients should have one core criteria from the following:

1. Involvement of optic nerve, spinal cord, dorsal medulla, brain stem, diencephalon, or cerebrum.

2. Positivity for Aquaporin-4.

3. Rule out mimics.

Aquaporin-4 testing is not available in most low-income countries which could be an obstacle for accurate diagnosis of NMOSD. Aquaporin- 4 antibody could be detected by any of the following methods:

1. Indirect immunofluorescence (IIF).

2. Enzyme-linked immunoassay (ELISA).

3. Cell based assay which can involve live cells or a commercial kit coated with pre-fixed cells.

4. (FACS) Fluorocytometry) using free floating live cells.

Cell based assay is quite sensitive and specific but is expensive, time consuming and requires expertise. Meanwhile, FACS is able to provide a quantitative assay [2].

Citation: Adel Ekladious. "New Trend in Neuromyelitis Optica Spectrum Disorder (NMOSD)”. Medicon Medical Sciences 2.3 (2022): 15-20. 


\section{Differential diagnosis and discussion \\ Multiple sclerosis (MS)}

In MS, the optic fiber layer wall is thickened in the temporal quadrant in contrast to NMOSD, where optic nerve layers are thickened in the superior and inferior quadrants. This can be confirmed by optical coherence tomography (OCT). Callosal lesions are small and not swollen as compared to NMOSD, where callosal lesions are large and quite edematous. MS tends to return positive serology results for measles, rubella and varicella, which is not the case for NMOSD. Furthermore, optic chiasma involvement is rare in MS but common in NMOSD [3]. Other findings prevalent and specific to MS are Dawson fingers and lesions adjacent to lateral ventricle and inferior temporal lobe. In MS, monoclonal bands are very common and do not disappear, while for NMOSD, oligoclonal bands are not common and usually disappear after treatment. With regards to symptoms, NMOSD presents with interactive hiccups, and nausea in cases of lesions in the area postrema, which are not apparent in MS. Extensive transverse myelitis with involvement more than three vertebrae in length, is a core feature found in NMOSD, not found in MS [4].

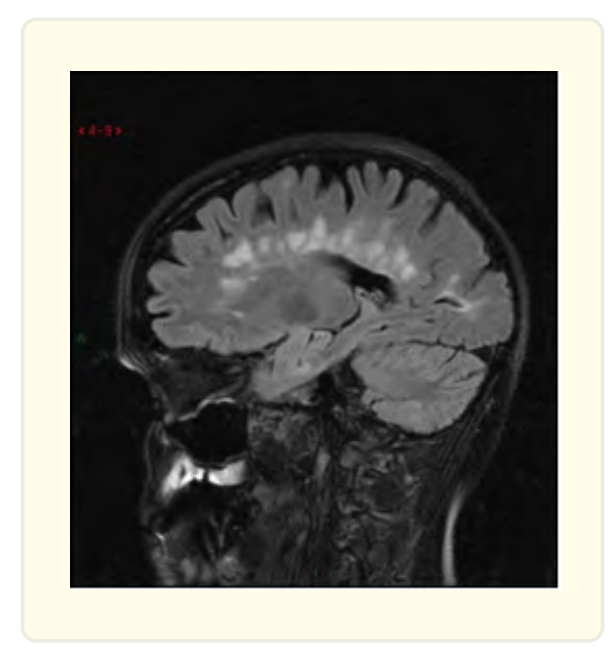

\section{Acute disseminated encephalomyelitis}

This disease commonly affects children. It is a polyfocal monophasic disease, preceded by vaccination or viral illness. This is usually associated with encephalopathy, where lesions in the hypothalamus favor the diagnosis. MRI usually shows lesions in the same stage of the disease. As an imaging modality, MRI is of paramount importance as it reveals lesions in the cord or the brain. Perineural enhancement has a high specificity for Myelin oligodendrocyte glycoprotein Antibody (MOG-AB). This is considered part of the NMOSD negative aquaporin-4 spectrum, which may develop extensive transverse myelitis and bilateral optic neuritis in the future [5].

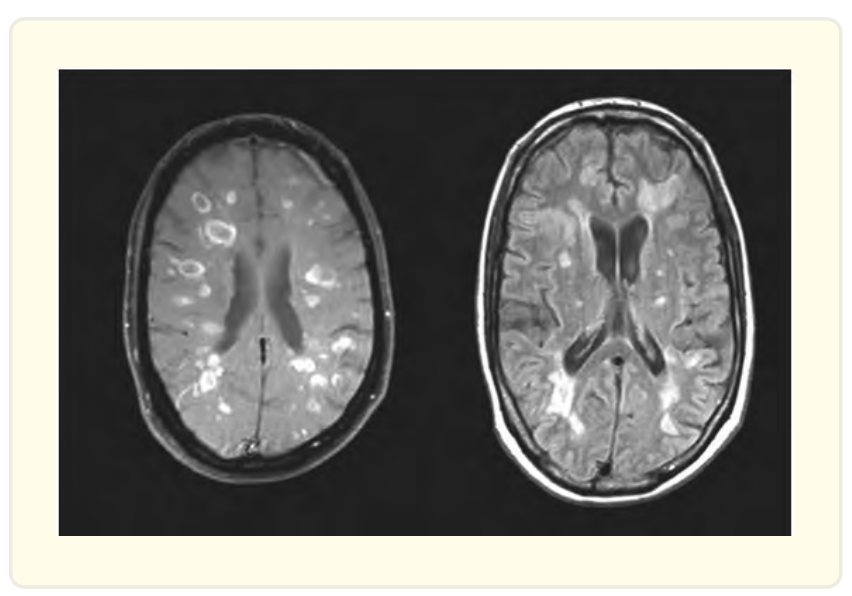

Citation: Adel Ekladious. “New Trend in Neuromyelitis Optica Spectrum Disorder (NMOSD)”. Medicon Medical Sciences 2.3 (2022): 15-20. 
Idiopathic optic neuritis

Idiopathic optic neuritis is a diagnosis of exclusion after ruling out all other differentials.

\section{Neurosarcoidosis}

Neurosarcoidosis is a disease that can affect the skin, lymph nodes, lung, heart, pituitary gland, in addition to causing optic neuritis and extensive transverse myelitis. FDG - PET Scan is a standard investigation for neurosarcoidosis. Findings include non-enhancing brain lesions and elevation of angiotensin converting enzyme Titer, Liver involvement, such as the development of granulomatous hepatitis, is common. Histopathology of non-caseating granuloma of the liver or skin is diagnostic of neurosarcoidosis [6-7].

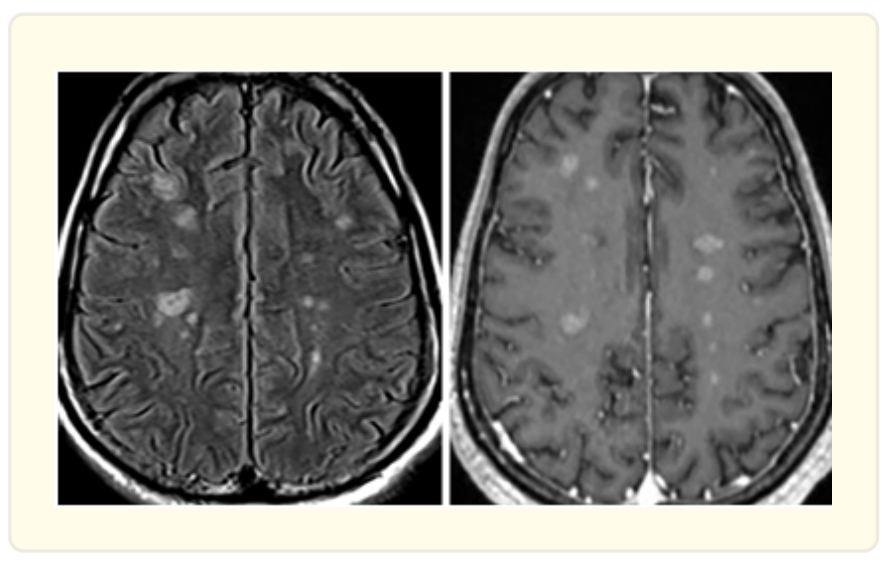

\section{Sjogren Syndrome}

Sjogren syndrome is a multisystem disease that can affect any organ in the body. Distinctive features include dry eyes and mouth. A positive Shermer's test and rose Bengal test are indicative of the disease, with a definitive diagnosis made through lymph node biopsy. Positive serology Anti-Ro, Anti-La, and Anti-Sim antibodies may also contribute towards a diagnosis of Sjogren syndrome. Similar to Aquaporin-4 positive NMOSD, patients with Sjogren syndrome may present with optic neuritis. Patients with no neurological involvement almost always test negative for Aquaporin-4 [8]. Thus, this could be interpreted as either the patient having coexisting NMOSD and Sjogren's, or that positive indicators for Aquaporin-4 are limited to patients who have Sjogren disease with neurological involvement.

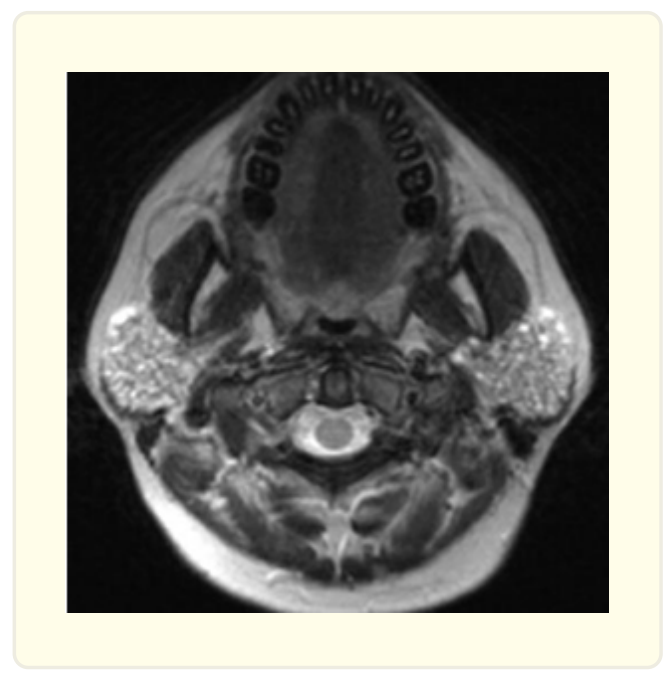




\section{Systemic Lupus Erythematosus (SLE)}

SLE is an autoimmune multisystem disease that can affect white and gray matter of the brain, causing paresis, ataxia, seizures, myelitis, optic neuritis, and nephritis. SLE may have lung and heart involvement as well. Serology tests for SLE are specific, and would present as a negative result for Aquaporin-4.

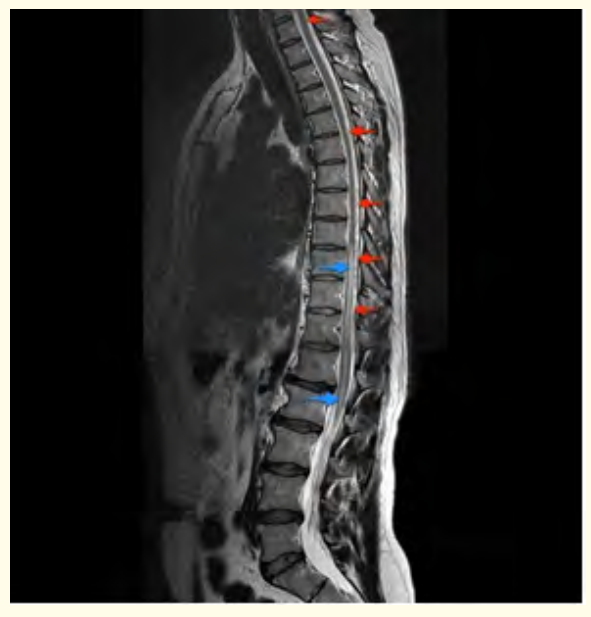

\section{Spinal Dural Arteriovenous fistula}

Spinal dural arteriovenous fistula is a disease that is predominantly found in males. It leads to decreased cord perfusion as a result of reduced arterio-venous pressure gradient. Progression of this disease can cause extensive transverse myelitis, with MRI usually showing extensive T2 hyperintensity. Other possible findings include abnormally dilated intra-dural veins, mainly in the dorsal cord. Hence, early diagnosis is essential as patients can be cured with surgical closure of the fistula and endovascular embolization [9-10].

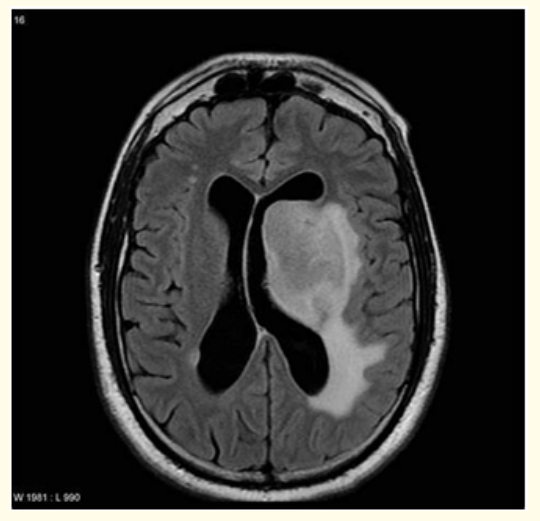

\section{CNS Lymphoma}

Patients with CNS lymphoma can develop very similar manifestations to NMOSD. FDG-PET scan plays an important role of investigation with stereotactic histopathology. Early steroid administration will mask the diagnosis and should not be given before confirming before a diagnosis is made [11]. 


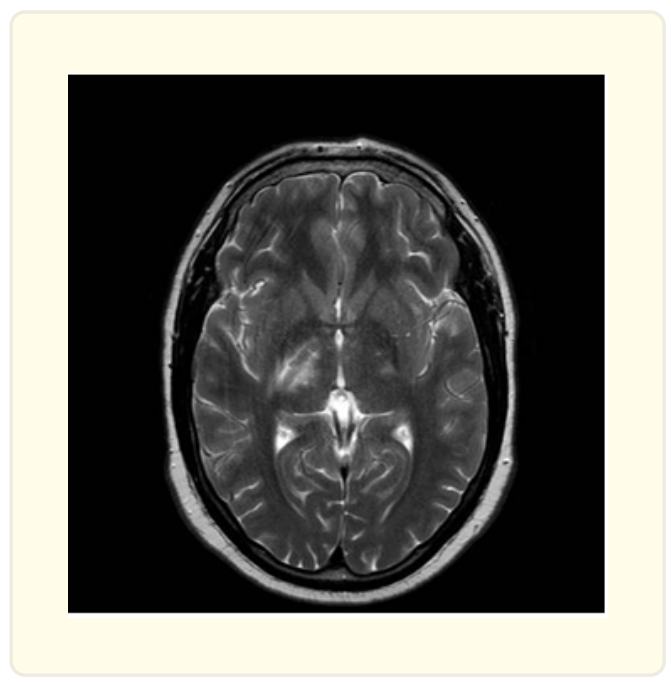

\section{Neuro-Bechet's disease}

Neuro-Bechet disease is an autoimmune multisystem condition common in the Middle East that typically presents as painful mucocutaneous ulceration [12]. It is known to affect arteries and veins, and can affect any area of the brain. Characteristic features of Bechet's disease are meningoencephalitis, severe atrophy of the brainstem and cerebrum. A positive pathergy skin test is commonly found in patients with Bechet's disease, and HLA-B51 genotype positivity usually confirms the diagnosis [13-15].

\section{Lebre Hereditary optic atrophy}

This is a mitochondrial disease that affects mainly males. It has been shown to have a strong familial link. Lebre hereditary optic atrophy causes bilateral simultaneous or consecutive optic atrophy, resulting in a progressive and painless loss of vision. There is an absence of gadolinium enhancement on MRI. This condition has a grave prognosis with no current treatment available [16].

\section{Infections}

TB, syphilis, Epstein Barr virus, Cytomegalovirus, Human T-lymphotropic virus, Mycoplasma pneumonia, Dengue virus. Infections with these pathogens present with a similar manifestation to NMOSD.

\section{Acknowledgment}

Professor Adel Thanks Mike Zhang (medical student Melbourne University) for editing the article and adding the Figures.

\section{References}

1. Miyazawa I, Fujhara K and itoyama Y. “Eugene devic (1858-1930)". J Neurol 249.3 (2002): 351-2.

2. Wingerchuk DM, Hogancamp WF, O Brien PC and weinshenker BG. "The clinical course of neuromyelitis optica (Devics Syndrome)". Neurology 53.5 (1999): 1107-14.

3. Wingerchuk DM, Lennon VA, Lucchinetti CF, PittockSJ and Weinshenker BG. "The spectrum of neuromyelitis optica". Lancet Neurol 6.9 (2007): 805-15.

4. Wingerchuk DM., et al. "International Panel for NMO Diagnosis, International Consensus diagnostic criteria for Neuromyelitis Optica Spectrum disorders". Neurology 85.2 (2015): 177-89.

5. Hung JS, Bhat RV, Preston GM, Guggino WB, Baraban JM and agre P. “Molecular Charcterization of an aquaporin c DNA from brain: 
Candidate Osmoreceptor and regulator of water balance". Proc Natl Acad Sci USA 91.26 (1994): 13052-6.

6. Papadopoulos MC and Verkman AS. "Acquaporin 4 and neuromyelitisoptica”. Lancet Neurol 11.6 (2012): 535-44.

7. Marrie RA., et al. "Dramatically changing rates and reasons for hospitalizations in multiple schelrosis", Neurology 83.10 (2014): 929-937.

8. Lassmann H, Van Horssen J and Mahad D. "Progressive multiple sclerosis pathology and pathogenesis". Nat Rev Neurol 8.11 (2012): 647-656.

9. Bevan RJ., et al. "Meningeal inflammation and cortical demyelnation in acute multiple sclerosis". Ann Neurol 84.6 (2018): 829842 .

10. Alves JM, Marques IB and Gil-Gouveia R. “[Vaccination controversies: An adult case of post Vaccinal acute Disseminated Encephalopmyelitis]". acta Med port 32.1 (2019): 81-85.

11. kawanaka Y., et al. "Delayed appearance of transient hyperintensity foci on T1- weighted magnetic resonance in acute disseminated encephalomyelitis". Jpn J Radiol 37.4 (2019): 277-282.

12. Gerke AK and Hunninghake G. "The immunology of sarcoidosis". clin. Chest. Med 29.3 (2008): 379-90.

13. Marangoni S, Argentiero V and Tavolato B. "Neurosarciodosis. Clinical description of 7 cases with a proposal of new diagnostic strategy". J Neurol 4 (2006): 488-95.

14. Alexander EL., et al. "Primary Sjogren syndrome with cenral nervous disease mimicking multiple sclerosis". Ann Intern Med 104.3 (1986): 323-330.

15. Mori K., et al. "The wide spectrum of clinicalmanifistations in Sjorgen syndrome associated neuropathy". Brain 128 (2005): 2534.

16. Hanly JG., et al. "Neuropsychatric events at the time of diagnosis of Systemic lupus erthromatosis An International inspection cohort study". Arthitis Rheum 56.1 (2007): 265-273.

Volume 2 Issue 3 March 2022

(C) All rights are reserved by Adel Ekladious. 